\title{
Mechanisms of the innate immunity in the respiratory system
}

\author{
KATARZYNA GRZELA', WIOLETTA ZAGÓRSKA ${ }^{l}$, TOMASZ GRZELA ${ }^{2}$ \\ ${ }^{1}$ Department of Paediatrics, Pneumonology and Allergology, Medical University of Warsaw, Poland \\ ${ }^{2}$ Department of Histology and Embryology, Medical University of Warsaw, Poland
}

\begin{abstract}
The respiratory system is permanently exposed to the influence of numerous harmful environmental factors: various microbial pathogens, allergens and air pollution, with non-volatile toxic chemicals of tobacco smoke. Therefore, there have been developed several mechanisms protecting from these factors. These mechanisms can be classified as innate, non-specific, or acquired, much more specific, which are associated with regular immune response. In this review authors shortly describe the innate, nonspecific mechanisms, which compose the first line of antimicrobial defense in our respiratory system. The mechanisms of innate immunity include passive protection, provided by the mucus-mediated mechanical barrier. Moreover, by means of active defense, several potent immunoregulatory factors and various substances displaying antibacterial, antifungal and antiviral activity are also engaged.
\end{abstract}

Key words: antimicrobial peptides, ciliated epithelium, innate immunity, mucus, respiratory tract.

(Centr Eur J Immunol 2012; 37 (3): 280-285)

\section{Introduction}

The respiratory tract is, next to the skin and the gastrointestinal tract, the third system of our body, which has permanent contact with dangerous environmental factors. These factors include numerous pathogenic bacteria, fungi and viruses, allergens or various chemicals of air pollution, with highly toxic, non-volatile components of tobacco smoke [1]. The exposure of the respiratory system to mentioned detrimental factors obviously depends on the level of the environmental pollution. However, in majority of urban areas with their usual concentration of the most common particulate matters (with the particle size above $10 \mu \mathrm{m}$ ) and respirable suspended particles (with the size below $10 \mu \mathrm{m}$ ), the pollutant load in respiratory tract may easily reach up to 0.5 milligrams of dry mass per day [2]. This amount of pathogens requires the presence of highly efficient system, which protects from the injury caused by those factors. Therefore, as a result of the evolution several protective mechanisms have been developed. These mechanisms are usually classified into two groups. The first group, known as the innate immunity, is represented by non-specific mechanisms. They involve passive protection provided by mechanical mucus barrier and mucociliary clearance, as well as active protection, mediated by numerous antimicrobial factors produced by epithelium and leukocytes [3]. The second group of protective mechanisms is classified as acquired immunity. It is much more specific, and is associated with processing and presentation of antigen by specialized cells, and its recognition by T lymphocytes, followed by an effector phase of immune response [4].

It is noteworthy, that mechanisms of innate immunity in respiratory system, although considered as more primitive, than acquired immune response, nevertheless they are quite composed (Fig. 1). Therefore, in this review authors would like to focus on the mentioned first line of our defense system.

\section{Mucus}

The main component of innate immunity in respiratory tract is the mechanical barrier. It protects from invasion of microbial pathogens or uncontrolled intake and exposure of organism to foreign antigens/allergens, which are inhaled into the respiratory tract together with the air. The first constituent of this protective barrier is thick mucous film, which overlays the second component of the barrier, formed by the pseudostratifed ciliated columnar epithelium. 


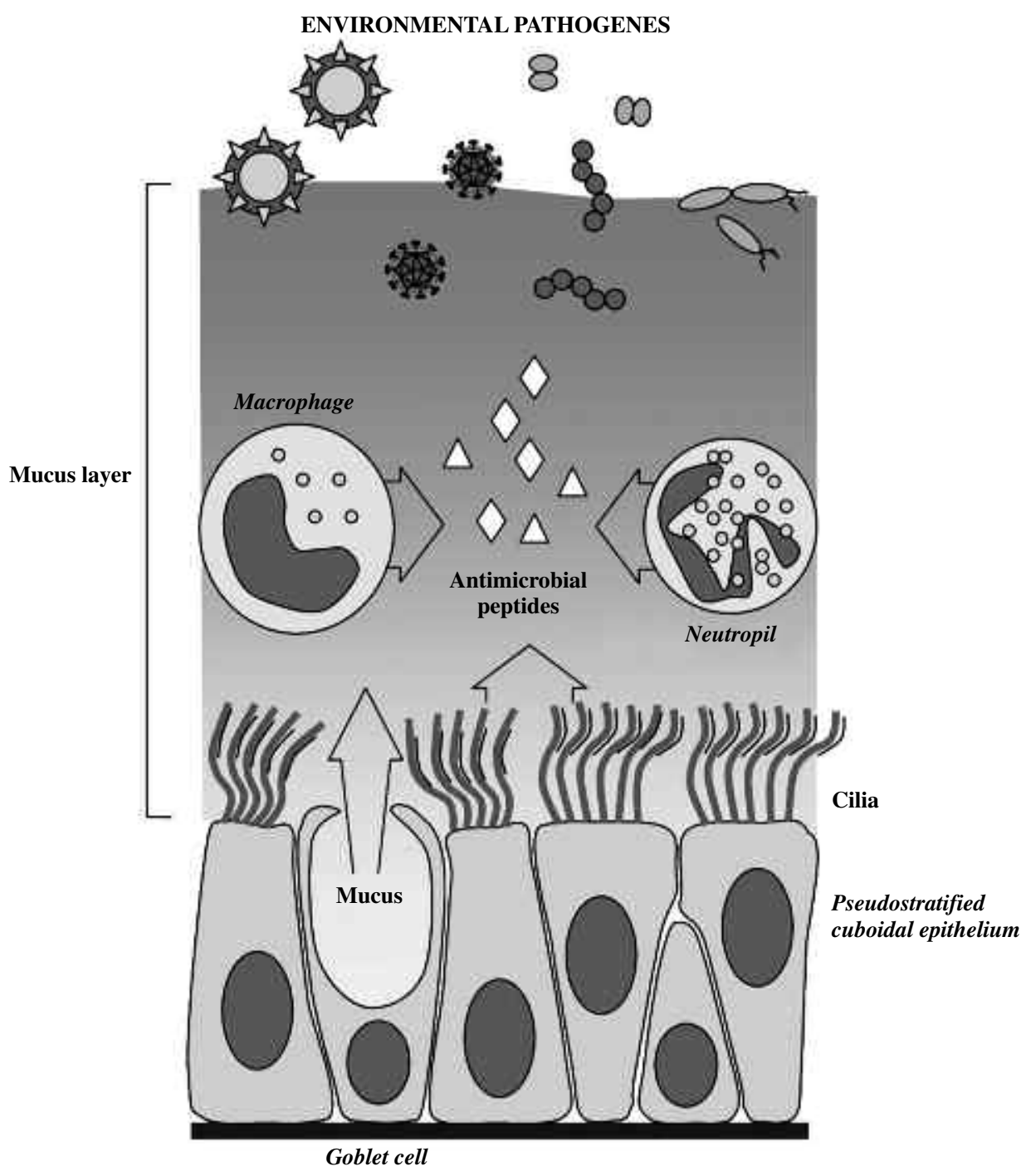

Fig. 1. The schematic representation of main mechanisms of the innate immunity in airway system (see detailed description in text)

\section{Mechanical barrier}

The mucus is a colorless, viscous and elastic gel-like substance, produced by single mucous cells, known as goblet cells, or by cell clustered in mucosal glands. Based on the mechanical and biochemical properties there are two distinguishable layers of mucus. The upper layer, which is more dense and viscous, plays a role of the trap or filter with the mesh of $100 \mathrm{~nm}$, responsible for immobilization of microbial cells and foreign particles [5]. When immobilized, those pathogens and particles are removed from the respiratory tract together with mucus towards the oral cavity. This mechanism is commonly known as the mucociliary clearance.

The upper layer of the mucus is composed mainly of large amounts of mucins, water and ions. Mucins belong to the family of at least 19 glycoproteins with the high molecular weight and extraordinarily high glycosylation level, that results in their high water-binding capacity and increased resistance to proteolysis [6]. Furthermore, cysteine-rich amine- and carboxy-termini of mucin molecules participate in formation of disulfide bonds within the same molecule, or between various monomers. Thus, disulfide bonds and non-covalent interactions between mucin molecules allow their arrangement into mega aggregates with molecular mass reaching much more than ten Mega Daltons (MDa) [7]. Majority of mucin monomers are secreted as a free component of mucus, however, some of them, e.g. MUC1, due to the presence of hydrophobic transmembrane domain may also be anchored in the cell membrane. There are data suggesting that these membrane-attached mucins may play some role in signal transduction [8]. Moreover, these transmembrane forms are also supposed to suppress the immune response against mucin-expressing cancer cells $[9,10]$. 


\section{Antimicrobial factors}

Besides a mechanical and chemical protection the mucus also provides the proper hydratation of the epithelium. This property is due to its thin hydrous layer, located between upper viscous mucus layer and epithelial cells [7]. The lower layer of mucus is produced by submucosal glands. When compared to the upper layer, it is less viscous, contains mainly water, ions and large quantities of various antibacterial/bactericidal substances, secreted by epithelial cells, neutrophils and macrophages. Among mentioned direct and indirect antimicrobial agents, the most important include: lysozyme, lactoferrin, secretory leukocyte proteinase inhibitor (SLPI), phospholipase A2, defensins, cathelicidins and surfactant proteins $[3,11,12]$.

\section{Lysozyme}

Lysozyme, also known as muramidase, or $N$-acetylmuramide glycanhydrolase, is one of main antibacterial factors of mucus. Its large quantities were detected in sputum (up to $1 \mathrm{mg} / \mathrm{ml}$ ), it can also be found in tears and serum $[13,14]$. It is produced by epithelial cells, but also has been found in azurophilic and specific granules of neutrophils, monocytes and macrophages. Lysozyme reveals the strong bactericidal activity, directed mainly against Gramm-positive bacteria. The mechanism of action is based on destruction of bacterial wall. It is due to lysozyme-catalyzed hydrolysis of 1,4- $\beta$ linkage between $N$-acetylmuramic acid and $\mathrm{N}$-acetylglucosamine in peptidoglycans, or between $\mathrm{N}$-acetylglucosamine residues in chitodextrins [14]. It has been demonstrated that lysozyme reveals synergistic effect with other antimicrobial factors - lactoferrin, SLPI and cathelicidins [15].

\section{Lactoferrin}

Lactoferrin, or lactotransferrin, is a globular glycoprotein, produced by epithelial exocrine cells; also it is present in specific granules of neutrophils [12, 14]. Lactoferrin was firstly discovered in milk, especially in human colostrum (so called "first milk"), where it reaches extraordinarily high concentrations (up to $7 \mathrm{~g} / \mathrm{l}$ ), however, its large quantities are also present in saliva and sputum (up to $1 \mathrm{mg} / \mathrm{ml}$ ), tears, serum and sperm [14]. The best recognized antibacterial effect of lactoferrin depends on chelating of iron cations $\left(\mathrm{Fe}^{3+}\right)$, which are necessary for bacteria metabolism and growth. Furthermore, lactoferrin can bind to bacterial or fungal wall and affects cell membrane permeability, possibly by formation of peroxides, thus leading to cell lysis $[16,17]$. Interestingly, other, iron chelating-independent mechanisms of its antimicrobial activity, were also reported [17]. It has been demonstrated that lactoferrin revealed an antiviral activity against a broad spectrum of pathogenic viruses, including: herpes simplex viruses 1 and 2, human immunodeficiency virus (HIV), hepatitis C virus (HCV), cytomegalovirus (CMV), rotaviruses, human respiratory syn- cytial virus (HRSV) and many others [18]. There are two main mechanisms of antiviral lactoferrin activity reported to date. First mechanism depends on competitive binding of lactoferrin to the same cellular lipoproteins, as these recognized by viruses on target cells. Alternatively, lactoferrin can directly bind to, and neutralizes viral particles. The second mechanism of antiviral activity is due to significant affinity of lactoferrin to nucleic acids, and its ribonuclease activity. Thus, lactoferrin seems to play an important role in protection, especially against retroviral infections [18].

\section{Secretory leukocyte protease inhibitor}

Secretory leukocyte protease inhibitor (SLPI), is cationic protein, which is secreted by epithelial cells of submucosal glands and surface epithelia. Its significant amounts are detectable in saliva, bronchial and nasal fluid. In nasal mucus its concentration reaches approximately $80 \mu \mathrm{g} / \mathrm{ml}$ [19]. The main role of this factor is to protect epithelia from endogenous serine proteases, e.g. neutrophil elastase, cathepsin G, trypsin, and mast cell chymase [20]. Therefore, the administration of recombinant human SLPI is considered as highly beneficial in reducing symptoms of cystic fibrosis, emphysema or asthma [19-21]. Moreover, SLPI also reveals some antimicrobial activity against a broad spectrum of microorganisms. In vitro studies revealed its effectiveness against Staphylococcus aureus, Pseudomonas aeruginosa, Escherichia coli, Klebsiella pneumoniae, Haemophilus influenzae, Streptococcus pneumoniae, Branhamella catarrhalis, and Aspergillus fumigatus, Candida albicans or Mycobacterium tuberculosis [21]. Recently, the protective role of SLPI was also postulated in viral infections, including its highly promising anti-HIV properties [22].

\section{Phospholipase A2}

Phospholipase A2 (PLA2), especially its secreted form (sPLA2) plays a key role in regulation of the immune response in the respiratory system [23, 24]. A main source of sPLA2 is the population of activated alveolar macrophages, however, in addition to macrophages, also the epithelial cells along the respiratory tract may produce sPLA2. The expression of SPLA2 may be induced by proinflammatory cytokines (e.g. tumor necrosis factor), or bacterial products, e.g. lipopolysaccharide (LPS), therefore an increased expression of sPLA2 is found mainly in areas of intense inflammatory reaction $[23,24]$. The main activity of SPLA2 concerns the generation of bioactive eicosanoids, stimulation of chemokines and cytokines expression, however, the direct bactericidal activity of sPLA2 against various bacteria was also reported [25].

\section{Defensins}

Defensins are arginine-rich, small cationic microbicidal peptides, which have been implicated in antibacterial and antifungal resistance $[16,26]$. The defensins bind to 
and damage microbial cell membranes by formation of porelike membrane defects. Thus, they result in target cell death by uncontrolled efflux of ions and nutrients. Defensins, especially two of them - the human $\beta$ defensin-1 (HBD-1) and -2 (HBD-2), play a key role in protection of human epithelia against microbial colonization. It has been found that HBD-1 is expressed constitutively, whereas the expression of HBD-2 molecule is induced by bacterial products/derivatives and by pro-inflammatory cytokines, mainly IL- $1 \beta$ and TNF $[3,11]$. In addition to their main membrane-associated activity, HBD-1 and -2 molecules may also reveal properties of chemotactic and activating factors for phagocytes - monocytes, and neutrophils, but also for dendritic cells and $\mathrm{T}$ lymphocytes, thus providing a link between innate and acquired immunity [11]. Moreover, it was reported, that defensins may neutralize some bacterial toxins, activate the complement pathway thus supporting the humoral immune response and display an antiviral activity, including some anti-HIV properties [14, 26-28].

\section{Cathelicidins}

Cathelicidins are a family of antimicrobial peptides, which are produced and stored in lysosomal granules of activated macrophages, neutrophils and epithelial cells [28]. The expression of cathelicidins is induced by bacteria, fungi, viruses, and by 1,25-dihydroxycholecalciferol, the active form of vitamin $\mathrm{D}_{3}[28,29]$. Interestingly, it has been reported, that cathelicidins may be involved in antituberculosis resistance. Furthermore, they may inactivate bacterial lipopolysaccharide and therefore reveal some protective activity against a development of septic shock [30]. Most recently, some synthetic analogs of cathelicidins are tested for their ability to treat infections caused by biofilm-forming bacteria strains, especially Pseudomonas aeruginosa [3].

\section{Surfactant proteins}

In addition to mentioned above, a crucial role in the innate immunity in the lowest parts of respiratory tract and in alveoli may play colectins: the surfactant proteins SP-A and SP-D [3, 11]. These proteins are able to recognize and bind to oligosaccharides, which are present on the surface of various bacteria. Such complexes are then recognized through the receptor for $\mathrm{C} 1 \mathrm{q}$ complement factor. Thus, the SP-A and SP-D induce phagocytosis and stimulate bactericidal properties of pulmonary macrophages $[3,11]$.

\section{Epithelium}

As previously described, the entrapment of microbial and dust particles is considered to reflect the main protective role of mucus. This mechanism however requires functional mucocilary clearance, which depends on a proper composition and consistence of mucus, but also is based considerably on the coordinated movement of epithelial cilia [31]. Their impaired movement, known as ciliary dyskinesia, may result in accumulation of dense mucus with attached highly concentrated bacteria, and, thus, it significantly increases the risk of recurrent infections of respiratory tract [32]. Furthermore, ciliary dyskinesia, especially its primary form, which also affects the reproductive system, may be responsible for ectopic fetus implantation and male infertility [33].

\section{Cilia}

The normal epithelial cells are represented mainly by cuboidal cells with 50-200 cilia located on their apical surface. The cilia are 5-7 $\mu \mathrm{m}$ long, with $0.2-0.3 \mu \mathrm{m}$ of diameter. Each cilium is composed of plasma membrane, which covers a core known as the axoneme. The axoneme is formed by the central pair of microtubules surrounded by 9 dublets of microtubules with attached dynein arms and other associated proteins involved in cilia movement $[34,35]$. Normal cilia reveal characteristic, coordinated, two-phase movement with mean frequency of approx. $12 \mathrm{~Hz}$ that enables the mucus transport with the average speed above $7 \mathrm{~mm}$ per minute $(>42 \mathrm{~cm} / \mathrm{h})[36,37]$. However, any changes, inherited or acquired, in structure and function of axoneme, including abnormal number and location of microtubules, or impaired function of microtubule-associated proteins - especially dynein arms, may result in abnormal movement of cilia and lead to disturbances in mucociliary clearance $[37,38]$. Based on the origin of mentioned abnormalities, the inherited, genetically determined changes are classified as primary ciliary diskinesia (PCD), whereas the acquired, usually associated with various detrimental factors (e.g. infection, allergy, or chronic exposure to tobacco smoke) are characterized as secondary ciliary dyskinesia (SCD) [37-41].

\section{Toll-like receptors and cytokines}

In addition to the previously described mechanical activity of the epithelial cells, they also express various factors involved in both, direct and indirect regulation of immune response. Among them there are Toll-like receptors (TLR) and various cytokines $[3,11]$. The members of TLR family are transmembrane glycoproteins, located on the cell surface, or intracellularly, in endosomes [42]. They are responsible for the recognition of pathogen-associated molecular pattern (PAMP), the conserved structures of various microorganisms, which activate the intracellular signaling pathways, and support pro-inflammatory response [43]. The TLR molecules mainly involved in pro-inflammatory response in respiratory system are TLR-2, -4 and -9 [44, 45]. The in vitro studies have shown that stimulation of TLR-2 and -4 increases the various cytokines production, including IL-1, -5, -6, -8, and GM-CSF by epithelial cells. They further stimulate the antimicrobial and antimycotic activity of last line of innate immunity, the professional phagocytes - macrophages, neutrophils and eosinophils, as well as engage components of acquired immune response $[3,11,46]$. 


\section{Concluding remarks}

The innate immunity in the respiratory tract involves three main mechanisms. The first component is mechanical barrier formed by upper layer of mucus. The second are various highly effective antimicrobial substances secreted to lower layer of mucus. The third mechanism is efficient mucociliary clearance. In addition, epithelial cells are able to express various immunoregulatory factors, including cytokines or TLR molecules, which further support immune response. Although considered as primary, or even primitive and unspecific, the mentioned mechanisms, if functioning well, are highly effective, whereas any disturbances in their function may lead to chronic or recurrent infections.

\section{References}

1. Curtis L, Rea W, Smith-Wallis P, et al. (2006): Adverse health effects of outdoor air pollutants. Environment International 3: 815-830.

2. WHO web page http://www.who.int/phe/health_topics/outdoorair/databases/en/

3. Ooi E, Psaltis AJ, Witterick I, et al. (2010): Innate immunity. Otolaryngol Clin N Am 43: 473-487.

4. Walker JA, McKenzie A (2012): Innate lymphoid cells in the airways. Eur J Immunol 42: 1368-1374.

5. Voynow JA, Rubin BK (2009): Mucins, mucus, and sputum. Chest 135: 505-512.

6. Evans CM, Koo JS (2009): Airway mucus: the good, the bad, the sticky. Pharmacology \& Therapeutics 121: 332-348.

7. Fahy JV, Dickey BF (2010): Airway Mucus Function and Dysfunction. N Engl J Med 363: 2233-2247.

8. Kim KC, Lillehoj EP (2008): MUC1 mucin: a pacemaker in the lung. Am J Respir Cell Mol Biol 39: 644-647.

9. Hollingsword MA, Swanson BJ (2004): Mucins in cancer: protection and control of the cell surface. Nat Rev Cancer 4: 45-60.

10. Taylor-Papadimitriou J, Burchel JM, Plunkett T, et al. (2002): MUC1 and the immunobiology of cancer. J Mammary Gland Biol Neoplasia 7: 209-221.

11. Proud D, Leigh R (2011): Epithelial cells and airway diseases. Immunol Rev 242: 186-204.

12. Parker D, Prince A (2011): Innate immunity in the respiratory epithelium. Am J Respir Cell Mol Biol 45: 189-201.

13. Parameswaran GI, Sethi S, Murphy TF (2011): Effects of bacterial infection on airway antimicrobial peptides and proteins in COPD. Chest 140: 611-617.

14. Lee HY, Andalibi A, Webster P, et al. (2004): Antimicrobial activity of innate immune molecules against Streptococcus pneumoniae, Moraxella catarrhalis and nontypeable Haemophilus influenzae. BMC Infect Dis 4: 12.

15. van der Linden DS, Short D, Dittmann A, Yu PL (2009): Synergistic effects of ovine-derived cathelicidins and other antimicrobials against Escherichia coli O157:H7 and Staphylococcus aureus 1056 MRSA. Biotechnol Lett 31: 1265-1267.

16. Scott MG, Hancock RE (2000): Cationic antimicrobial peptides and their multifunctional role in the immune system. Crit Rev Immunol 20: 407-431.

17. Farnaud S, Evans RW (2003): Lactoferrin - a multifunctional protein with antimicrobial properties. Mol Immunol 40: 395-405.
18. van der Strate BW, Beljaars L, Molema G, et al. (2001): Antiviral activities of lactoferrin. Antiviral Res 52: 225-239.

19. Singh PK, Tack BF, McCray PB Jr, Welsh MJ (2000): Synergistic and additive killing by antimicrobial factors found in human airway surface liquid. Am J Physiol Lung Cell Mol Physiol 279: L799-L805.

20. Żbikowska-Gotz M, Pałgan K, Socha E, et al. (2011): Measurement of effector properties of neutrophilic granulocytes in patients with allergic hypersensitivity to food. Post Dermatol Alergol 28: 175-180.

21. Sallenave JM (2010): Secretory leukocyte protease inhibitor and elafin/trappin-2. Versatile mucosal antimicrobials and regulators of immunity. Am J Respir Cell Mol Biol 42: 635-643.

22. Wahl SM, McNeely TB, Janoff EN, et al. (1997): Secretory leukocyte protease inhibitor (SLPI) in mucosal fluids inhibits HIV-1. Oral Dis 3: S64-S69.

23. De Luca D, Minucci A, Cogo P, et al. (2011): Secretory phospholipase A2 pathway during pediatric acute respiratory distress syndrome: a preliminary study. Pediatr Crit Care Med 12: 20-24.

24. Henderson WR Jr, Oslund RC, Bollinger JG, et al. (2011): Blockade of human group X secreted phospholipase A2 (GXsPLA2)-induced airway inflammation and hyperresponsiveness in a mouse asthma model by a selective GX-sPLA2 inhibitor. J Biol Chem 286: 28049-28055.

25. Nevalainen TJ, Graham GG, Scott KF (2008): Antibacterial actions of secreted phospholipases A2. Review. Biochim Biophys Acta 1781: 1-9.

26. Lee SH, Kim JE, Lim HH, et al. (2002): Antimicrobial defensin peptides of the human nasal mucosa. Ann Otol Rhinol Laryngol 111: 135-141.

27. Münk C, Wei G, Yang OO, et al. (2003): The theta-defensin, retrocyclin, inhibits HIV-1 entry. AIDS Res Hum Retroviruses 19: 875-81.

28. De Y, Chen Q, Schmidt AP, et al. (2000): LL-37, the neutrophil granule- and epithelial cell-derived cathelicidin, utilizes formyl peptide receptor-like 1 (FPRL1) as a receptor to chemoattract human peripheral blood neutrophils, monocytes, and T cells. J Exp Med 192: 1069-1074.

29. Doss M, White MR, Tecle T, Hartshorn KL (2010): Human defensins and LL-37 in mucosal immunity. J. Leukoc Biol 87: 79-92.

30. Golec M (2007): Cathelicidin LL-37: LPS-neutralizing, pleiotropic peptide. Ann Agric Environ Med 14: 1-4.

31. Houtmeyers E, Gosselink R, Gayan-Ramirez G, et al. (1999): Regulation of mucociliary clearance in health and disease. Eur Respir J 13: 1177-1188.

32. Jorissen M, Bertrand B, Eloy P (1997): Ciliary dyskinesia in the nose and paranasal sinuses. Acta Otorhinolaryngol Belg 51: 353-366.

33. Afzelius BA (2004): Cilia-related diseases. J Pathol 204: 470-477.

34. Fliegauf M, Benzing T, Omran H (2007): When cilia go bad: cilia defects and ciliopathies. Nat Rev Mol Cell Biol 8: 880-893.

35. Marshall W (2008): The cell biological basis of ciliary disease. J Cell Biol 180: 17-21.

36. Salathe M. (2007): Regulation of mammalian ciliary beating. Annu Rev Physiol 69: 401-422.

37. Jorissen M (1998): Correlation among mucociliary transport, ciliary function and ciliary structure. Am J Rhinol 12: 53-58.

38. Armengot M, Milara J, Mata M, et al. (2010): Cilia motility and structure in primary and secondary ciliary dyskinesia. Am J Rhinol Allergy 24: 175-180. 
39. Leopold PL, O'Mahony MJ, Lian XJ, et al. (2009): Smoking is associated with shortened airway cilia. PLoS ONE 4: e8157.

40. Tamashiro E, Xiong G, Anselmo-Lima WT, et al. (2009): Cigarette smoke exposure impairs respiratory epithelial ciliogenesis. Am J Rhinol Allergy 23: 117-122.

41. Verra F, Escudier E, Lebargy F, et al. (1995): Ciliary abnormalities in bronchial epithelium of smokers, ex-smokers, and nonsmokers. Am J Respir Crit Care Med 151: 630-634.

42. Kumar H, Kawai T, Akira S (2009): Pathogen recognition in the innate immune response. Biochem J 420: 1-16.

43. Lazarczyk M, Grzela K, Grzela T (2005): Immunostimulatory oligonucleotides in therapy of allergic diseases. Expert Opin Biol Ther 5: 525-536.

44. Dong Z, Yang Z, Wang C (2005): Expression of TLR2 and TLR4 messenger RNA in the epithelial cells of the nasal airway. Am J Rhinol 19: 236-239.

45. Tan L, Rogers TJ, Hatzirodos N, et al. (2009): Immunomodulatory effect of cytosine-phosphate-guanosine $(\mathrm{CpG})$-oligonucleotides in nonasthmatic chronic rhinosinusitis: an explant model. Am J Rhinol Allergy 23: 123-129.

46. Pitzurra L, Bellocchio S, Nocentini A, et al. (2004): Antifungal immune reactivity in nasal polyposis. Infect Immun 72: 7275-7281. 